\title{
Robespierre et la liberté des noirs en l'an II d'après les archives des comités et les papiers de la commission Courtois
}

Jean-Daniel Piquet

\section{(2) OpenEdition \\ Journals \\ Édition électronique \\ URL : https://journals.openedition.org/ahrf/1822 \\ DOI : $10.4000 /$ ahrf.1822 \\ ISSN : 1952-403X \\ Éditeur : \\ Armand Colin, Société des études robespierristes}

Édition imprimée

Date de publication : 1 mars 2001

Pagination : 69-91

ISSN : 0003-4436

\section{Référence électronique}

Jean-Daniel Piquet, «Robespierre et la liberté des noirs en l'an II d'après les archives des comités et les papiers de la commission Courtois ", Annales historiques de la Révolution française [En ligne], 323 | janvier-mars 2001, mis en ligne le 16 mars 2006, consulté le 22 avril 2022. URL : http:// journals.openedition.org/ahrf/1822 ; DOI : https://doi.org/10.4000/ahrf.1822

Ce document a été généré automatiquement le 22 avril 2022.

Tous droits réservés 


\title{
Robespierre et la liberté des noirs en l'an II d'après les archives des comités et les papiers de la commission Courtois
}

\author{
Jean-Daniel Piquet
}

Le 16 pluviôse an II (4 février 1794), l'esclavage était aboli pour la première fois dans le monde. La spécificité de cette abolition ne réside pas seulement dans son caractère pionnier mais aussi dans un contexte idéologique très particulier: un pays en révolution, une colonie en insurrection et un mouvement populaire très influent sous l'autorité de Maximilien Robespierre. Le rôle de ce dernier, qui représente l'osmose entre le mouvement populaire et la défense universelle des Droits de l'homme de 1789 reste à éclaircir et synthétiser. Sa chute en juillet 1794 et les polémiques qu'elle a engendrées ont brouillé la vision de ce personnage relativement à cette première abolition. Colons de Saint-Domingue et antiesclavagistes de la métropole se sont affrontés sur la mémoire (ou plutôt la contre-mémoire) du personnage: agent des trois députés de Saint-Domingue, porteurs de l'abolition après leur arrivée à Paris, ou complice des planteurs et résolument hostile à l'émancipation des Noirs. L'attitude de Robespierre sur cette question ne manque pas de contradictions et a suscité des doutes sur ses intentions supposées antiesclavagistes. Et pour cause, ses quasi-silences sur un décret qu'il aurait dû applaudir frénétiquement à la lumière de ses anciens combats ont alimenté une polémique dans laquelle les avocats de la thèse d'un révolutionnaire plutôt hostile à cette abolition dominent. Ainsi, selon Aimé Césaire, Robespierre, qui à la Constituante avait eu de belles paroles telles que « Périssent les colonies s'il doit vous en coûter votre honneur", non seulement se tut à la Convention (hormis une dénonciation de la traite négrière en avril 1793 que Césaire a oubliée alors que ses biographes l'avaient relevée), mais de plus apostropha, le 17 novembre 1793, «la faction Brissot» souhaitant à la fois "réduire le peuple au statut d'hilotes et le soumettre à l'aristocratie des riches, et en un instant affranchir et armer tous les nègres pour perdre nos colonies $»^{1}$. A.Césaire y voyait une confirmation de certains 
propos que lui a attribués en février 1795 l'un des trois députés de Saint-Domingue, Dufaÿ, se référant à Barère et faisant état d'une opposition de Robespierre à l'abolition antérieure au 16 pluviôse an II souhaitée au contraire par son collègue; l'auteur percevait aussi dans la démarche de Robespierre une démarche nationaliste de coexistence pacifique avec l'Angleterre ${ }^{2}$. Y.Bénot a repris cette thèse en soulignant davantage le nationalisme expansionniste qui exigeait le maintien du statu quo dans les colonies $^{3}$. C.Wanquet suppose que Robespierre s'inscrivait aussi dans le soutien à Camille Desmoulins auteur d'un pamphlet, Jean-Pierre Brissot démasqué en février $1792^{4}$. Mais l'auteur ne tranche pas entre les deux interprétations du nationalisme formulées par A. Césaire ou par Y. Bénot. Et, toujours selon lui, à l'exception de Collot d'Herbois, auteur de l'Almanach du père Gérard, instigateur de l'arrestation de Page et Brulley, l'abolitionnisme des Montagnards était pratiquement inexistant et leur silence a été particulièrement frappant dans l'attitude à l'égard des Mascareignes ${ }^{5}$. Note-t-on aussi : ce que Robespierre a dit en public sur les Girondins après leur élimination, il le répète peu avant leur arrestation contre Danton et Delacroix : «Danton m'a dit un jour : il est fâcheux que nous ne puissions céder nos colonies aux Américains. Depuis, Danton et Lacroix ont fait passer un décret dont les conséquences sont vraisemblablement la perte des colonies $»^{6}$. Un ami de Robespierre, Jeanbon Saint-André, passe pour être résolument esclavagiste. Outre de longues relations, elles certaines, avec Page et Brulley entre l'été 1793 et l'hiver 1794, un discours colonialiste tenu le 3 septembre 1793 où Saint-André présentait les Blancs comme des victimes des Noirs, de la politique émancipatrice de Sonthonax, ainsi qu'un document d'archives postérieur à l'abolition, ont accrédité cette image. À telpoint que si Y.Bénot admet la possibilité, en l'attente d'autres témoignages, de l'intervention de Robespierre en faveur de la pétition abolitionniste du 4juin 1793, signalée dans une affiche, au côté de l'abbé Grégoire, eu égard à ses interventions passées en mai 1791 et avril 1793 en ce qui concerne Jeanbon Saint-André également mentionné il l'exclut pratiquement ${ }^{7}$. Nous avons déjà mis en relief deux documents où Robespierre paraît étroitement lié dans sa politique en l'an II à la députation de Saint-Domingue ${ }^{8}$.

2 C.L.R. James et Florence Gauthier ont évoqué Robespierre et les problèmes coloniaux dans leurs relations avec le mouvement populaire. Pour le premier $\left({ }^{(9)}\right.$, Robespierre était un bourgeois, dépassé sur sa gauche, qui desservit la cause antiesclavagiste par son combat contre les Enragés et les Hébertistes. Pour la seconde -qui fait exception au groupe précité d'interprétations négatives de l'Incorruptible et de la question coloniale ${ }^{(10)}$ - Robespierre symbolisa tout au contraire la défense du droit naturel dans toute son étendue, aussi bien à la Constituante en 1791 qu'à la Convention : en avril 1793 il inséra la dénonciation de l'esclavage dans son projet populaire d'économie politique. Des manipulations expliqueraient les propos de novembre 1793, notamment les dénonciations constantes de Sonthonax, Polverel et du diplomate Genêt par les colons blancs de Saint-Domingue présents à Paris.

3 À la lecture de documents d'archives inédits nous devons renoncer à l'interprétation d'un groupe robespierriste hostile ou indifférent à l'abolition ou d'une exception géographique quelconque dans la volonté de liberté et d'égalité. Deux auteurs ont discuté des relations de Robespierre avec Danton: Georges Hardy, Michelet. Nous ne pouvons pas dire au contraire de ce que croyait le premier que Danton et Delacroix s'étaient désintéressés des questions d'émancipation coloniale, ni que Danton avait suivi Barnave en mai 1791. Michelet, est l'unique historien de la Révolution au 
XIxesiècle à avoir évoqué -fût-ce de manière elliptique- le 16 pluviôse an II (4 février 1794) et ses suites, adresses et fêtes, quoi qu'on en ait dit par ailleurs. Mais il a souligné le rôle de Danton sans percevoir la continuité d'une action abolitionniste montagnarde après sa mort en avril 1794 jusqu'à la fin juillet 1794 en corrélation avec le mouvement populaire. Les formes complémentaires de l'action robespierriste se sont harmonisées dans les relations pouvoir/mouvement populaire, le second devançant parfois le premier, et méritent d'être analysées dans le sens vu par F.Gauthier mais au travers de documents inédits. Le récent travail de C.Wanquet fournit des témoignages étayant celui trouvé par Y.Bénot, relativement aux soutiens reçus par Grégoire le 4juin 1793 au sein de la Convention.

4 À l'inverse, les multiples explications retenant soit les dérapages antibrissotins de l'automne 1793, soit les convictions esclavagistes, soit le nationalisme, soit la manipulation, soit le combat contre le mouvement populaire nous paraissent sinon fausses du moins simplificatrices. Des documents extraits des papiers de la commission Courtois, des archives des Comités de salut public, de sûreté générale ou des colonies montrent en fait que Robespierre se situait formellement ou tacitement dans l'action abolitionniste de l'anII dont les registres ou les décrets comptaient autant que les propos de 1791 (lesquels avaient leur importance) et les ont même radicalisés. La relation Desmoulins-Brissot-Robespierre de 1792 constitue une contre-preuve d'un dérapage alors étranger à Robespierre qui nous le verrons mais s'est davantage démenti en 1793 et $1794^{11}$. Par ailleurs, Aimé Césaire a assez gravement déformé les propos de Barère et Dufaÿ qui, après Thermidor, n'ont pas osé attribuer à leur ancien collègue un passé esclavagiste. Mais nous ne partageons pas tout à fait l'interprétation des manipulations à propos de la phrase du 17 novembre 1793 considérant que dans le cadre des choix politiques de la Montagne, il y a bien eu déphasage robespierriste avec une partie du mouvement populaire après le 4 juin 1793; il y a aussi dans le mouvement lui-même, la désunion du groupe des libres de couleur, plus aussi unanimement antiesclavagiste qu'en juin 1793 comme il y a une certaine dislocation du groupe des colons blancs. Ces réalités brouilleront la vision des questions coloniales par Robespierre qu'il comprendra mieux après l'arrivée des trois députés de SaintDomingue et le décret du 16 pluviôse an II(4 février 1794).

Prélude à l'abolition : la pétition du 4 juin 1793 et ses enjeux politiques

5 Les journées des 31 mai, 1er et 2 juin 1793 sont connues pour être celles de l'expulsion de 29 députés girondins de la Convention. Ce à quoi on ajoutera dans les semaines suivantes l'incarcération de 73 autres députés brissotins qui protestèrent contre ces expulsions. Beaucoup d'entre eux étant d'anciens membres de la Société des Amis des Noirs. Ce rapprochement a fait dire à certains que l'antiesclavagisme n'avait désormais plus cours dans la grande Assemblée républicaine. Pourtant, ces journées sont marquées par la mobilisation de la Commune, des Jacobins et de la Montagne à la Convention pour faire aboutir l'abolition de l'esclavage dans les colonies ${ }^{12}$. Robespierre s'insère dans ce mouvement aux Jacobins comme à la Convention. Le 3 juin le Club des Jacobins accueillit en son sein des hommes de couleur en présence de Robespierre et engagea une discussion sur lesmodalités d'intégration au club des nouveaux arrivants. Le 4 juin 1793 une délégation de sans-culottes et d'hommes de couleur, conduite par Chaumette, présente à la Convention une pétition demandant la liberté générale des Noirs dans les colonies et un drapeau tricolore représentant les trois couleurs des colonies enfin réconciliées ${ }^{13}$. Dans l'Assemblée le mouvement est soutenu non seulement par l'abbé Grégoire mais aussi, selon le témoignage d'un métis de la 
Martinique présent, Julien Labuissonnière, par Robespierre, Jeanbon Saint-André (également présent le 3 juin aux Jacobins) et d'autres «justes » de la Montagne dont ferait partie Couthon d'après un correspondant de Labuissonnière, Milscent. D'après Labuissonnière, ces membres de la "Sainte » Montagne échouèrent du fait des "vils campagnards de la Convention $»^{14}$. Une semaine plus tard, ledit Labuissonnière est inculpé pour vol et le 11 juin J.-F.Varlet, militant « enragé » de la Commune, y dénonce un complot blanc destiné à faire échouer la pétition. Un grand mouvement pour l'abolition immédiate de l'esclavage se structurait. Les trois noms des personnalités montagnardes considérées généralement soit comme relativement indifférentes (Couthon, Robespierre) soit comme hostiles (Saint-André) à l'abolition, le contenu de l'intervention d'un «Enragé » indiquent la portée et les limites de ce mouvement. Ces interventions témoignent d'un intérêt de la Montagne pour cette abolition malgré (ou à cause de) la chute de la Gironde. D'autres témoignages que celui de Labuissonnière existent: ceux des milieux colons des Mascareignes ${ }^{15}$. En juillet 1793 l'un d'eux rapporte avec inquiétude qu'une bonne partie de la Convention approuve la pétition, et un autre que Grégoire paraît désormais prudent. Par ailleurs même s'il est vrai que les accusations contre Labuissonnière ne provenaient pas forcément d'un complot $\left({ }^{16}\right)$, la réaction de Varlet témoigne de la part d'une bonne partie du mouvement populaire à l'égard des aristocrates de Saint-Domingue d'une solidarité avec leurs frères sansculottes noirs des colonies et d'une haine de leurs bourreaux blancs.

De ce fait, une partie de la Montagne se mit sous l'influence de véritables intrigants. On pense notamment à la phrase de Robespierre prononcée le 17 novembre 1793 opposant les droits à la liberté générale des Noirs au maintien des colonies. Ce jacobin avancé sembla opposer les droits des pauvres en métropole et le droit à la liberté des esclaves. Ni la thèse des convictions esclavagistes du personnage, de la volte-face pour alliance avec Danton, ni celle de la manipulation pure et simple ne sauraient convenir. L'affaire du 4 juin 1793 est déjà significative : les plaintes de Labuissonnière sur les tentatives des députés du Marais d'étouffer la revendication abolitionniste de "la Sainte Montagne» doivent être prises en compte. Car ils constituaient une partie non négligeable des auditeurs de Robespierre le 17novembre. Mais ils ne sont pas les seuls.

7 Mais il faut aussi citer la double dislocation du groupe de pression des Blancs et des hommes de couleur, au second semestre 1793.

8 Le premier point est caractérisé par l'emprisonnement de Philippe-Rose Roume, le 27 juin 1793, ancien commissaire à Saint-Domingue sur dénonciation à Rochefort de Victor Hugues ${ }^{17}$. Bien qu'ami de Marat, Roume, ancien gouverneur de Tabago, ne partageait pas en 1791 sa sympathie pour la cause des Noirs, esclaves ou mulâtres. Il avait alors défendu le maintien des prérogatives des assemblées coloniales de SaintDomingue $^{18}$. Mais il fut malgré tout nommé commissaire pour porter le décret du 15 mai 1791 en faveur de l'égalité des Blancs et des libres de couleur citoyens actifs non affranchis. À peine apprit-il à Saint-Domingue la révocation de ce décret le 24 septembre, qu'il appela les métis à se rendre et à se fier à la générosité des Blancs prêts à leur pardonner. Il dut cependant fléchir devant les victoires militaires des mulâtres déterminés à ne pas se laisser faire (comme l'avait prophétisé Marat en septembre 1791). Aussi progressivement en deux ans Roume modifia-t-il son point de vue dans un sens plus progressiste au point d'être considéré comme traître par nombre de colons (Hugues, Page, Brulley). Il n'avait cependant pas évolué sur le droit à la liberté et à l'égalité des esclaves. De retour à Paris, début 1793, du fond de sa prison en juillet il 
s'adressa une première fois à son ami Marat qui accepta de prendre sa défense au tribunal révolutionnaire (et qui venait le 6 juillet d'être élu au comité colonial de la Convention $\left.\left.{ }^{[19}\right]\right)$, juste avant d'être poignardé dans les conditions que l'on sait. Roume adressa alors à Danton et Robespierre une lettre d'explication sur les événements de Saint-Domingue. Il fut libéré en août sur initiative d'un ami de Danton, Chabot, qui percevait cet emprisonnement comme "une trame» des colons blancs ${ }^{20}$. Mais Roume fut aussi relaxé grâce au témoignage à décharge au tribunal révolutionnaire, d'un colon, Larchevesque-Thibault ${ }^{21}$ qui malgré son amitié pour Page et Brulley fit preuve du même pragmatisme que lui à l'égard des droits des mulâtres. On trouve dans les papiers de Danton le rapport de Roume du 27 janvier 1793, accompagné d'une pièce de Larchevesque-Thibault :

«On ne peut plus dire désormais que ce soient les citoyens de couleur et nègres libres qui fomentent, qui attirent la rébellion de nos esclaves; ils n'ont plus d'intérêt à l'entretenir; ils ont au contraire le même intérêt que nous à la faire cesser.

Il y a donc une cause sourde qui travaille nos esclaves. Quelle est-elle cette cause, si ce n'est la fureur d'opérer, à quelque prix que ce soit la contre-révolution?

Je veux croire que la philanthropie entre pour beaucoup dans les malheurs que nous éprouvons ; mais cette philanthropie n'y entre que comme instrument et non comme cause. Il fallait aux contre-révolutionnaires un appât qu'ils pussent présenter à nos esclaves pour les soulever contre la servitude ; ils l'ont trouvé dans la Déclaration des droits de l'homme. Mais sans les contre-révolutionnaires jamais cette déclaration n'eût produit sur nos esclaves une impression assez forte pour les faire passer tout d'un coup de la soumission la plus parfaite aux derniers excès de la révolte. $»^{22}$

9 Cette pièce assimile la Déclaration des droits de l'homme, sur laquelle s'appuient les esclaves, à un complot de la Contre-Révolution. Elle exclut de l'intrigue les libres de couleur. Robespierre, allié de Danton, s'est fondé en toute bonne foi sur le texte d'une personne qui a dénoncé une trame pour en faire passer une autre.

Il faut aussi compter avec l'entrée à la Convention du premier libre de couleur, Janvier Littée, député de la Martinique, en septembre $1793^{23}$. Il s'agit comme Labuissonnière d'un ayant droit à la loi du 4 avril 1792 (accordant définitivement, du moins jusqu'en 1802, l'égalité des Blancs et des libres de couleur sans discrimination aucune), mais qui au contraire de lui est comme Larchevesque-Thibault très lié à Page et Brulley, dont il partage les vues d'ensemble sur la situation à Saint-Domingue. En la présence des deux colons, le 12 juillet 1793, Littée rencontra Jeanbon Saint-André24. Le 3septembre ce dernier tourna complètement le dos à l'intervention rapportée par Labuisssonière du 4 juin 1793: "Les événements malheureux dont la ville du Cap a été le théâtre et la victime depuis le 19 juin jusqu'au 23 sans interruption; ces événements, dont est résulté l'assassinat des Blancs, qui ont forcé une quantité de marchands d'abandonner cette rade et de se réfugier dans les États-Unis ont été l'ouvrage de Polverel et de Sonthonax $»^{25}$. Ce discours colonialiste où les colons blancs esclavagistes réunis autour de Galbaud, contre les hommes de couleur et Noirs alliés à Sonthonax et Polverel étaient présentés comme ses victimes, a pu être prononcé sous l'influence de Page et Brulley certes, mais aussi sous celle du «nouveau citoyen » de couleur Janvier Littée. Cette allocution ne signifie donc pas forcément une opposition de principe à l'émancipation des Noirs ni même un reniement.

11 Cela est encore plus vrai de Robespierre. Car quand il s'exprima devant la Convention le 17 novembre, ce «nouveau citoyen » de couleur, convaincu de la nocivité du principe 
de la liberté générale des esclaves, était député depuis le 19 septembre 1793. À ce sujet lorsque Garran-Coulon affirme l'opposition probable de Robespierre à la liberté générale des esclaves, il confirme notre conjecture. À l'appui de son propos, Garran fournit deux références différentes, qui l'une et l'autre commentent la phrase du discours de novembre 1793, la première au travers de Littée, la seconde de Page et Brulley. La première provient d'un pamphlet thermidorien de Leborgne, créole antiesclavagiste de la Martinique contre Littée : Leborgne y accuse Littée d'appartenir à une faction qui « inspira l'infâme discours de Robespierre contre la liberté des Noirs " ${ }^{(26)}$.La seconde référence, Garran-Coulon l'appelle « Registre de la commission de SaintDomingue, fin pluviôse »: soit le cahier de Page et Brulley. Or pour l'ensemble de la période concernée, 17-30 pluviôse (5-17 février 1794), aucune réaction de Robespierre à l'abolition n'y filtre. En revanche on trouve dans le registre, à la « fin brumaire » deux réactions satisfaites de Page et Brulley au « discours lumineux de Robespierre $»^{27}$.

On peut certes considérer la phrase comme une preuve indirecte de cette opposition et l'expliquer par un nationalisme outrancier peu rationnel, qui voudrait maintenir les colonies telles quelles afin de ne pas y jeter un trouble susceptible d'être exploité par les ennemis ou rivaux de la France. Le nom de Camille Desmoulins, auteur d'un violent pamphlet contre Brissot en 1792 et allié de Robespierre dans l'opposition à la guerre, vient à l'esprit. En outre, en décembre 1793 les deux Montagnards s'unissent une dernière fois dans ce qu'on commence à appeler la crise des factions, contre Cloots et n'ont pas de mots assez forts pour fustiger « l'étranger ", « le prussien ${ }^{28}$. Ne serait-ce pas la preuve d'un dangereux tournant xénophobe?

13 Il faut cependant se souvenir que lorsque Robespierre s'opposa en 1792 avec acharnement au bellicisme des Girondins, un mois après la déclaration de guerre du 20 avril, il se livra à un bilan plutôt négatif de l'action des Girondins à l'Assemblée législative en faveur des libertés, mais remarqua avec ostentation un unique élément positif: le combat zélé des Brissotins pour l'égalité des Blancs et des libres de couleur qui aboutit à la loi du 4avril $1792^{29}$. Robespierre désavouait ainsi Desmoulins qui, en février 1792, dans son pamphlet Jean-Pierre Brissot démasqué, amalgamait en Brissot l'humanisme de Las Casas et l'expansionnisme d'Alexandre le Grand. Camille a dit que les 2 premiers numéros du Vieux Cordelier avaient été contrôlés par Robespierre avant leur parution ${ }^{30}$. Ses écrits de haine fielleuse à l'encontre de Cloots le 10 décembre 1793, relayés oralement par Robespierre le 12, sont plus complexes qu'il n'y paraît. Car n'oubliant pas la question coloniale, Camille tourne le dos à Jean-Pierre Brissot démasqué, et rejoint le Robespierre de 1791 et 1792 :

"Quoiqu'il ait des entrailles de père pour tous les hommes, Cloots semble en avoir moins pour les nègres ; car dans le temps, il combattait pour Barnave contre Brissot dans l'affaire des colonies; ce qui montre une flexibilité de principes et une prédilection pour les Blancs peu digne de l'ambassadeur du genre humain. En revanche on ne peut donner trop d'éloges à son zèle infatigable à prêcher la république une et indivisible des quatre coins du monde, à sa ferveur de missionnaire jacobin à vouloir guillotiner les tyrans de Chine et du Monotapa. Il n'a jamais manqué de dater ses lettres depuis cinq ans, de Paris, chef-lieu du globe ; et ce n'est pas sa faute si les rois de Danemark, de Suède gardent leur neutralité, et ne s'indignent pas que Paris se dise orgueilleusement la métropole de Stockholm et de Copenhague. $»^{31}$

14 Camille Desmoulins articulait ainsi l'universalité des principes de la Révolution au refus d'exporter par les armes les principes de liberté aux quatre coins du monde. Si Robespierre a bien participé à la rédaction de l'article, cela montre qu'il maintient ses 
positions sur la question mulâtre, alors que Desmoulins paraît s'être singulièrement métamorphosé par cet hommage implicite à Brissot. Comme en 1792, pour Robespierre et Camille Desmoulins, la guerre de l'an II reste une guerre défensive ${ }^{32}$. Qu'en est-il pour Robespierre de la traite et de l'esclavage ? Les deux problèmes se retrouvent à cette même époque, en janvier 1794, dans les inédits de la commission Courtois de 1824 et ont à ce titre été censurés par le célèbre Conventionnel. Un Jacobin anglais, Patterson, résidant à Boulogne-sur-mer, le 4 pluviôse an II (23 janvier 1794) rendait hommage à Robespierre pour son discours du 21 nivôse an II (10 janvier 1794) où pour souder le club fissuré par la crise des factions, il appelait à se préoccuper des «vices de la Constitution anglaise », parmi lesquels « un des plus importants » :

«Il y a environ deux ans que tout le peuple anglais exprimait formellement et constitutionnellement son vœu contre la traite des nègres. La nation entière dégoûtée des horreurs commises dans cet infernal commerce, la nation entière pétitionnait. Accablé de cette unanimité l'exécrable Pitt semblait céder au désir du peuple. Cependant la Chambre des Communes, dirigée secrètement par ce traître, osait remettre à quatre ans l'espérance de la nation... mais ô honte cette espérance échouait totalement dans la Chambre des Pairs. ${ }^{33}$

Patterson se souvenait du discours de Robespierre sur les Droits de l'homme du 24 avril 1793 qui déboucha sur la suppression des primes à la traite, votée le 27 juillet 1793 sur proposition de l'abbé Grégoire, au moment où l'Incorruptible entrait au Comité de salut public. Ce point amena sans doute Courtois à censurer la lettre ; mais aussi censura-t-il la partie relative au droit des gens :

«(...) que sur ses débris s'élève l'autel de la liberté et de l'égalité où la France et l'Angleterre puissent jurer une paix constante, une fraternité sans réserve. Voilà mes plus ardents souhaits et en t'exprimant ma reconnaissance pour la discussion que tu as ouverte à cet égard je ne suis pas sans savoir combien elle est capable d'amener leur accomplissement. Je suis bien convaincu qu'une voie est maintenant ouverte par laquelle la vérité peut entrer triomphante dans notre île. Il me reste à désirer que cette voie soit aplanie et gardée par une sagesse égale à celle qui a su la montrer. Empêche cher Robespierre, je te le conjure, qu'on y jette les pierres de la discorde. Les épithètes injurieuses que l'on prodigue à notre caractère national et individuel ne sont-elles pas pourtant des obstacles non moins fatals aux progrès de la vérité ? Tu sais bien que tout peuple est essentiellement bon, même celui auquel j'appartiens. » (souligné dans le texte)

16 Patterson souhaite faire tomber la constitution anglaise comme Robespierre, et en appelle à son autorité modératrice et à son esprit universaliste de fraternité de celui-ci. Il a à l'esprit « la perfide Albion » parfois employée mais absente des discours les plus violents de Robespierre qui se limitent aux attaques contre la Monarchie corrompue et contre les hommes politiques anglais qui l'acceptent. Pour Patterson la paix pourrait s'établir entre les deux peuples quand les Anglais, prenant conscience de l'injustice de leur combat, renverseront leur constitution aristocratique, que les Français de leur côté sous l'impulsion de Robespierre arrêteront les injures cocardières. L'image d'un Robespierre à la fois nationaliste et/ou esclavagiste en l'an II, qui de nos jours domine encore une grande partie de l'historiographie française, est démentie par ce document méconnu. En 1795 les risques politiques provoqués par un tel démenti ne sont pas sans expliquer la censure de la pièce par la Commission Courtois.

Il existe d'ailleurs un autre document colonial censuré par Courtois relatif à l'arrivée des trois députés de Saint-Domingue, en février 1794, à la Convention. Ces trois hommes symbolisaient les trois couleurs de l'ile, réconciliées: Dufaÿ (blanc), Mills (métis), Belley (noir) ${ }^{34}$. Ce document implique le mouvement populaire lorientais et 
deux Montagnards robespierristes : Jullien de Paris et Prieur de la Marne. Jullien de Paris envoie une lettre à Robespierre le 26 nivôse an II (15 janvier 1794) qu'il termine ainsi: «Je profite aujourd'hui pour t'écrire du départ de trois députés de SaintDomingue, près la Convention. Le citoyen Barbier, chargé par Prieur de les accompagner, est un patriote de Lorient qui doit faire plusieurs demandes pour la commune.Tu provoqueras l'adoption de celles qui te paraîtront justes. $»^{35}$

Il s'agit de la seule lettre de la correspondance Jullien-Robespierre, restée inédite en 1795.Ce document a donc été censuré. Il faut y voir le désir de camoufler la considération pour la députation de Saint-Domingue d'un homme de confiance de l'Incorruptible, en prison au moment de la publication du document, ce probablement par Garran-Coulon qui fit également supprimer sa propre lettre adressée à Carnot en juin 1794 dans laquelle il se félicitait de l'échec des attentats contre Robespierre et Collot d'Herbois ${ }^{36}$. On doit noter qu'après une première accusation par Leborgne en septembre 1794 contre Littée de complicité avec Robespierre, le député de la Martinique lui répliqua que Robespierre avait au contraire sauvé Leborgne d'une comparution devant le Tribunal révolutionnaire ${ }^{37}$. Or cette dernière imputation n'est pas dénuée de tout fondement : l'arrivée de Leborgne en France en novembre 1793 s'est déroulée de manière analogue à celle de Dufaÿ, Mills et Belley en janvier 1794. Du fond de sa prison le 29 janvier 1794, dans une lettre à la Convention, Leborgne oppose les intrigues dont il a été victime à son arrivée à Paris vers le 20 novembre 1793 à l'accueil fraternel qui lui fut accordé le 3 novembre par les protagonistes de Lorient : la Société populaire de la Montagne, Prieur et Jullien ${ }^{38}$.

La police de l'abolition jusqu'au plus haut niveau de l'État

19 Le décret du 16 pluviôse an II(4 février 1794) brille par son étendue et son originalité qu'aucun autre décret abolitionniste ne lui a disputées. On ne rappellera jamais assez qu'à la différence de tous les autres décrets d'émancipation négrière (dont celui de 1848), il refusait toute indemnité aux propriétaires. Le facteur révolutionnaire était au centre de ce refus: les colons sont relégués au rang de la noblesse et du clergé dépossédés sans condition en 1789 de leurs privilèges. En fait, loin d'avoir entrepris, comme les révolutionnaires de 1848 la moindre négociation avec les planteurs, les Conventionnels, parmi lesquels la plupart des membres du Comité de salut public, et/ ou les sections populaires ont séquestré nombre de leurs porte-parole présents à Paris. Faits qui s'inscrivent en faux à la fois contre la théorie d'un suivisme du mouvement populaire et contre celle d'une indifférence des Montagnards à laquelle Collot d'Herbois ferait exception.

Dans les débats entre les accusateurs et les accusés, Page et Brulley rappellent à Sonthonax et Polverel, qui leur reprochent d'avoir toujours trouvé bonne écoute auprès du Comité de salut public, leur arrestation quelques jours avant le 19 ventôse an II (9 mars 1794) sur un ordre de ce Comité. Ces dernières années, cet ordre a effectivement été retrouvé ; rédigé et signé par Collot d'Herbois et Saint-Just après réception par eux de deux lettres de dénonciation : une de Dufaÿ, Mills et Belley contre les activités occultes des deux hommes, une autre de Roume. Cet ordre dans lequel les deux lettres sont citées prouve les opinions et le rôle abolitionniste des deux Montagnards (et pas seulement comme l'écrit C.Wanquet du premier, Collot d'Herbois) ${ }^{39}$. Mais Page et Brulley ajoutent aussi avoir été précédemment arrêtés le 5 mars 1794 par la section des Tuileries. Le 4 mars Dufaÿ lui dénonça la Commission( ${ }^{40}$ ), et obtint immédiatement satisfaction. L'examen du registre quotidien de Page et Brulley corrobore au moins le 
récit de la datation de leurs premiers ennuis ce 4 mars. Ce même jour est celui du dernier récit de leur registre ${ }^{41}$.

21 Cette fois-ci Roume rompt avec l'analyse de Larchevesque-Thibault, à laquelle il avait sacrifié en 1793, d'une insurrection d'esclaves manipulée par la Contre-Révolution. Collot d'Herbois et Saint-Just tinrent compte du témoignage de Roume dans leur enquête pour la rédaction de l'ordre d'arrestation du 17 ventôse an II (7 mars 1794) de Page et Brulley; la police de l'abolition était en marche : il ne suffisait plus pour le Comité de salut public et la Convention d'être acquis à la cause des mulâtres pour être patriote ; il fallait aussi cesser de présenter l'insurrection d'esclaves comme manipulée par la Contre-Révolution. Dans cette période précise les Robespierristes jouent leur rôle, montrent le caractère unitaire du décret d'abolition. Les dossiers de police générale F7 4700 des colons indiquent qu'à la fin mars 1794 la nouvelle Commune robespierriste relaya la politique entamée par Chaumette et les Hébertistes peu avant leur élimination d'arrestations massives des membres d'assemblées coloniales, symboles vivants de l'aristocratie de la peau. Ce fait n'a jamais été introduit dans les études robespierristes des quatre derniers mois de l'an II dans les relations de Robespierre avec le mouvement populaire. Par exemple, pour raison de santé pendant quinze jours, du 10 au 24 mars 1794, Larchevesque-Thibault fut arrêté et gardé à domicile par un sans-culotte de la section de la Halle au Bled ${ }^{42}$. Dans la nuit du 24 au 25 mars, en pleine épuration des Hébertistes, la police de la Commune de Paris sévit et transféra Larchevesque-Thibault à la prison du Luxembourg. La cause de ce changement n'est pas politique mais financière : le coût financier important pour la section. Jusqu'à l'automne 1794 aucune réclamation de Larchevesque-Thibault et de sa femme ne firent changer d'avis les autorités ${ }^{43}$. Après thermidor, Page et Brulley n'eurent pas tort d'affirmer qu'au faite de sa puissance, au printemps et au premier mois de l'été 1794, Robespierre avait été l'un des plus puissants alliés des trois députés de Saint-Domingue. Il est fort possible à cet égard qu'ils aient participé activement à la fête de l'Être suprême à laquelle ils étaient de toute façon invités comme tous les autres députés. Un autre esclavagiste l'affirme. Le jour même, 20 prairial an II (8juin 1794), Thomas Millet, colon de Saint-Domingue incarcéré depuis six semaines à la prison des Carmes, s'indigne par lettre au Comité de salut public de la présence de Dufaÿ à "l'auguste fête ${ }^{44}$. Ce témoignage paraît crédible en raison de son détachement du contexte passionnel thermidorien. Pour répliquer au rapport présenté par Dufaÿ le 4 février 1794, relatif à une hostilité des colons blancs aux Noirs, Millet invoque au contraire l'alliance des colons blancs et des colons de couleur contre les esclaves à Saint-Domingue qu'il prétend prouver par un exemple personnel (une orpheline métisse qu'il a amenée à Paris dont les parents auraient été victimes d'un massacre de nègres) ${ }^{45}$.

22 Si Garran-Coulon a conclu à une opposition de Robespierre à la liberté générale et immédiate des esclaves en novembre 1793, des compléments d'information montrent qu'en juillet 1794 au moins, ses deux sources relatives au député de couleur, Janvier Littée et à Page et Brulley s'avèrent anachroniques.

23 Le soupçon existe certes que Robespierre ait fréquenté jusqu'en thermidor Page et Brulley. Si Y.Bénot affirme qu'aucune relation directe n'a existé entre eux, selon lui le premier a une fois reçu les deux intrigants le 4 février 1794 dans une séance du Comité de salut public. C.Wanquet va cependant plus loin en arguant de l'existence plausible de contacts personnels entre Robespierre et Brulley; il s'appuie sur des informations 
fournies en ce sens le 22 août 1794 par le montagnard thermidorien, Thuriot ${ }^{46}$. Pourtant les deux allégations sont sujettes à caution. L'une se fonde sur l'indication inexacte selon laquelle le 16 pluviôse an II (4 février 1794), à l'instar de Prieur de la Marne et de Saint-Just, Billaud-Varenne se trouvait en mission ${ }^{47}$. L'autre ignore la réponse de Page et Brulley : d'après eux il s'agissait d'un homonyme, "Brulé ", " envoyé en mission dans divers départements voisins de la Vendée lorsqu'on y faisait la guerre ${ }^{48}$.

En fait un document, cité par Page, nous indique ce qu'il en est des relations de Robespierre avec Janvier Littée et les deux colons de Saint-Domingue après l'abolition :

« Pour juger de l'influence dont nous jouissions, lisez le rapport Courtois. Vous y verrez que Littée, notre ami, le seul qui nous ait soutenu dans la Convention nationale, était poursuivi par les agents de Robespierre; et vous y verrez avec quelle précaution Robespierre se faisait rendre compte des numéros que nous adressions au Comité de salut public, intitulés: Notes au Comité de salut public. Si nous eussions été dans un si grand rapport avec le Comité de salut public, Robespierre n'aurait pas fait épier nos démarches, \& fait des recherches pour savoir ce que nous écrivions et ce que nous n'écrivions pas ; enfin il n'aurait pas fait suivre et espionner Littée, qui était notre protecteur dans la Convention. $»^{49}$

La référence à l'édition de 1795 des papiers Courtois sonne étrangement. On a vu que Courtois avait censuré deux papiers relatifs aux colonies; mais il affirmait en même temps n'en avoir trouvé aucun. De fait, sans le savoir, Courtois en a publié un que nous citons ici :

« Du 27 messidor an II :

Un des citoyens que nous occupons, a donné à dîner, le 20 messidor courant à la citoyenne Masse, et l'a conduite au bal. Ce fut le même jour qu'elle lui remit le $n^{\circ} 5$, et ils parlèrent peu de l'objet en question. Le même citoyen l'a revue chez elle le 24 et lui ayant dit qu'il avait oublié le $n^{\circ} 5$, elle l'engagea à le brûler sous peine d'être compromis puisqu'il ne devait être connu que de certains députés, et elle lui fit espérer le 6e numéro.

Littée donna à dîner, le 22 courant à cinq de ses collègues desquels sont les citoyens Las et Chesnier, qui engagèrent fortement la citoyenne Masse de demeurer avec le citoyen Littée; et sur l'observation qu'elle leur fit qu'elle serait compromise s'il arrivait quelqu'événement, on lui répondit : Nous nous entendrons pour vous faire une pension, si vous ne pouvez être dédommagée par le mobilier du citoyen Littée. La citoyenne Masse dit à notre agent que le citoyen Littée se faisait aimer de tous ceux qui le connaissaient, parce qu'il est franc et qu'il a un caractère prononcé contre l'injustice. Il a, dit-elle, des ennemis, mais qu'il ne craint pas. Le citoyen Littée est homme de couleur : il a épousé une femme très riche. Il a à Saint-PierreMartinique beaucoup de maisons et propriétés qui lui sont conservées par les Anglais par égard pour sa femme.

Il est certain qu'il existe un parti attaché au citoyen Littée; mais il faut d'autres renseignements pour fixer l'opinion qu'on doit en avoir. Nous osons espérer de parvenir à en découvrir la source, si notre homme peut conserver cette femme qu'infailliblement il perdrait, si elle allait demeurer avec le citoyen Littée, car elle est toujours très en garde sur les questions qu'il lui fait.

Comme on faisait espérer de donner, sous peu, le $\mathrm{n}^{\circ} 6$ nous avons d'autant plus la facilité de le faire aujourd'hui que nous avons découvert le dépôt ; il paraît que celui qui en est chargé doit distribuer ces écrits, puisqu'il les porte chez les différentes personnes qu'il croit faites pour les lire; et ce qui peut confirmer ce soupçon c'est que demain nous pouvons obtenir les numéros et la brochure que nous joignons ici. $\|^{50}$

26 Ce texte de Claude Guérin est daté du 15 juillet 1794, soit quatre mois après l'emprisonnement de Page et Brulley. Il explique que depuis au moins une semaine, cet 
agent s'enquérait des démarches de Littée. Elles lui étaient suspectes à deux titres : les relations avec les Anglais qui occupaient alors la Martinique et ses contacts avec les colons de Saint-Domingue symbolisés par les $n^{\circ} 5$ et $n^{\circ} 6$ des Notes fournies au Comité de salut public. Disons d'emblée que le $\mathrm{n}^{\circ} 6$ ne figure pas dans le recueil imprimé à la Bibliothèque nationale de France ${ }^{51}$. Sa parution prochaine était annoncée à la fin du volume $^{52}$. Les manuscrits ${ }^{53}$ ne correspondent qu'aux textes des cinq numéros imprimés et disponibles à la Bibliothèque nationale de France. En fait ce $n^{\circ} 6$ est celui dont Page $a$ cru bon de dire "que nous n'écrivions pas » par opposition au précédent que «nous écrivions ». Les deux sources utilisées par Garran-Coulon pour imputer à Robespierre une " opposition absolue » à l'abolition se retournent contre la théorie de son auteur. En juillet 1794, trois mois avant Leborgne, Robespierre considérait Littée comme un Contre-Révolutionnaire. Et dans ce crime de contre-révolution entraient les délits de diffamation-xénophobie-racisme à l'encontre de chacun des trois députés de SaintDomingue. Car, dans sa première partie, le $\mathrm{n}^{\circ} 5$ attaquait violemment la députation de Saint-Domingue : Dufaÿ y était traité « d'aristocrate », Mills « d'agent anglais », Belley "d'Africain Bambara». Le rapport de Guérin montre aussi que l'Incorruptible s'intéressait également aux moyens financiers (les pensions faites à la citoyenne au cas où elle "serait compromise ») destinés à encourager la diffusion de ce pamphlet aussi contraire aux idées universelles de liberté et d'égalité. Mais toute cette action eut lieu en osmose avec le mouvement populaire lequel se montra parfaitement autonome. Ainsi la section parisienne de Bonne-Nouvelle anticipa le décret du 9 mars $1794:$ le 27 pluviôse an II (15 février 1794), elle assigna à résidence deux de ses membres, colons de Saint-Domingue, au motif qu'ils avaient pétitionné contre la députation de SaintDomingue lors de son arrivée à Paris et fait opposition « au décret sur la liberté des hommes de couleur ». Jusqu'en messidor an II ces deux colons, Derragis et Derosière, subirent cette assignation. Mais, coup de théâtre, le 20 messidor (8 juillet 1794), l'un d'entre eux, Derosière, fut incarcéré à la prison des Carmes. D'après son propre témoignage, il devait ce transfert à un certain Jarry, porteur d'un ordre du Comité de salut public daté du 11 messidor (29juin) et signé Robespierre, Saint-Just, Couthon, Billaud-Varenne ${ }^{54}$. Si nous n'avons pas la preuve formelle de cet ordre, ainsi attribué aux plus radicaux des membres du Comité de salut public présents à Paris, son existence n'en est pas moins plausible. Billaud-Varenne connaissait fort bien Jarry : il reconnut publiquement en mai 1794 avoir recours à ses services pour faire suivre Tallien. Or après le 9 thermidor, il sera question du $n^{\circ} 5$ des notes de Page et Brulley dans les débats de la Convention qui nous indiquent le nom d'un de ses porteurs... Derragis. Comment ne pas y voir une relation avec l'ordre de transfert et d'incarcération de son ami Derosière? Les quatre signataires n'auraient-t-il pas cherché à faciliter les recherches de leurs agents en incarcérant l'ami de ce Derragis?

Un doublement des appointements à Claude Guérin a été décrété dans les premiers jours de thermidor par Couthon et Saint-Just. Mais cette surveillance de Littée fut peutêtre aussi encouragée par Jeanbon Saint-André, très souvent en mission dans l'ouest de la France. Ceci nous amène à réviser les idées reçues d'une hostilité de Jeanbon à l'abolition. Au travers du journal de Page et Brulley, nous n'avons aucune indication de leur part sur l'opinion de Jeanbon relative au décret du 16pluviôse. La seule information étayant son éventuelle hostilité active à l'abolition est inexacte et de toute façon sans grande portée $e^{55}$. Mais surtout dans l'ouest de la France, il mena lui aussi avec Prieur de la Marne une politique de neutralisation des ennemis de l'émancipation créant une commission sur les colonies et envoyant à ses collègues parisiens, par une 
lettre de juin 1794, une liste de colons de Saint-Domingue emprisonnés. Jeanbon qui, en septembre 1793, opposait les intérêts des sans-culottes français à ceux des esclaves noirs renverse la dialectique : les planteurs blancs sont des ennemis de la République au même titre que les émigrés européens. Or précisément il revint à Paris entre les 11 et 18 messidor (29 juin et 6 juillet 1794) juste au moment de l'ordre de transfert de Derragis, mais une semaine avant le début de la surveillance de Littée. Dans ses conversations avec ses collègues, Saint-André n'a-t-il pu alors discuter des questions coloniales déjà contenues dans sa lettre de début juin et par la même occasion signaler les liens de Littée avec Page et Brulley, qu'il avait rencontrés, tous les trois le 12juillet 1793 ?

Les décrets d'application du Comité de salut public et les problèmes politiques du moment

Le contexte de l'élimination physique de Danton et Delacroix a de fait accrédité l'idée d'un esclavagisme de Robespierre visible dans une signature à un décret d'accusation de Sonthonax et Polverel le 22 germinal anII (11avril 1794) qu'il n'a pas apposée à celui de l'abolition le 23 germinal anII (12 avril 1794). Paradoxalement, c'est une source à laquelle n'a pu avoir accès Garran-Coulon, qui crédibilise ses soupçons sur l'opposition de Robespierre et le complot esclavagiste antidantoniste : Les notes de Robespierre contre les Dantonistes ${ }^{56}$.

Robespierre relie le décret du 16 pluviôse an II à une offensive passée des Dantonistes à l'automne 1793 en faveur de la rétrocession des colonies antillaises à l'Amérique en échange d'une alliance militaire avec les États-Unis. Et cette phrase comme celle contre Brissot laisse percevoir une volonté de maintenir la politique de neutralité à l'égard des États-Unis. Cette puissance, il convenait diplomatiquement d'en ménager à la fois la neutralité et les structures sociales esclavagistes. Faut-il pour autant en conclure que Danton et Delacroix ont été guillotinés entre autre pour cette raison? En fait la phrase a été surestimée par quelques-uns de ses analystes $\left({ }^{57}\right)$, car, note A. Mathiez, Saint-Just qui a relu et corrigé les notes de Robespierre « laissa tomber l'observation $»^{58}$. Or au vu des liens qui existaient entre ces deux amis, comment ne pas croire que Saint-Just n'ait pas discuté de ce détail du rapport et n'ait alors raisonné son ami ? Robespierre était-il comme en novembre 1793 sous l'influence de Littée?

Michelet résume en une phrase la situation: "L'affranchissement des Noirs et les réactions d'ivresse et d'enthousiasme qu'il suscita attendrirent également les cœurs ». Outre que cette phrase montre un caractère moins complet de l'occultation qu'il n'a été dit, elle présente l'intérêt d'intégrer le thème de la liberté et de l'égalité des Noirs comme plus tard chez Jaurès l'égalité des sang-mêlé à la Constituante et à la Législative - dans une analyse politique : ici la rivalité Danton-Robespierre. Selon Michelet pour arracher aux Robespierristes le monopole des mesures de bienfaisance, les Dantonistes surenchérissent dans "les mesures d'attendrissement »: l'élévation - par la voix de Cambon- à 10millions de francs d'une somme de secours votée pour les indigents, somme ainsi vingt fois supérieure à celle de $500000 \mathrm{~F}$, proposée par les Robespierristes $^{59}$. Et si comme Michelet le dit « deux reines ne peuvent régner sur une même ruche " ${ }^{(60)}$, n'excluons pas qu'à ce titre l'une des deux, Robespierre, en qui ces mesures provoquaient l'alarme, en ait voulu à l'autre de cette surenchère sans qu'on puisse vraiment assurer qu'il la récusait.

31 Cependant quoi qu'en ait pensé Michelet "ces scènes d'ivresse et d'enthousiasme " continuèrent après la mort des Dantonistes. Robespierre n'a certes pas commenté le décret du 16 pluviôse an II notamment à la séance du 17 pluviôse an II (5 février 1794) 
où il aurait pu souligner l'immoralité de l'esclavage alors qu'une offensive se développait pour son maintien ${ }^{61}$. Mais le rapport d'un agent du Comité de sûreté générale, Latour-Lamontagne, juxtapose l'intervention de Robespierre au Club des jacobins le 19 pluviôse an II (7 février 1794) pour l'exclusion de Brichet et Santex qui réclamaient la comparution devant le Tribunal révolutionnaire des conventionnels de la Plaine, à un débat colonial assez original. «On faisait ce soir la motion d'abandonner aux hommes de couleur la partie de Saint-Domingue qui appartient aux Espagnols. Si ce décret était rendu disait l'orateur je répondrais de sa prompte exécution. Cette proposition a trouvé des approbateurs et des contradicteurs $»^{62}$. Une discussion a visiblement eu lieu sur le droit à l'autodétermination du peuple noir sous tutelle espagnole: intégration à la France de Santo-Domingo, ou indépendance de cette colonie espagnole libérée de la servitude esclavagiste. Nous ne connaissons malheureusement pas les noms de «l'orateur» qui formula cette proposition ou de ses «approbateurs et contradicteurs » faute d'avoir trouvé le moindre écho de ce débat dans la presse jacobine ${ }^{63}$. Ce silence s'explique-t-il par la crainte d'évoquer sur la place publique une question aussi délicate que l'indépendance d'une colonie ? Ou par celle de traiter d'un débat colonial auquel Robespierre participait et dont les propos risquaient selon l'hypothèse exprimée récemment par Claude Mazauric de susciter des polémiques inutiles ${ }^{64}$. L'unicité du témoignage privé de T.Millet sur le caractère antiesclavagiste de la fête parisienne de l'Être suprême peut aussi l'expliquer ; mais aussi à distance, l'écho dans la presse parisienne de l'antiesclavagisme de la fête brestoise de l'Être suprême, organisée par Prieur de la Marne :

«Un de ces hommes que la barbare politique de l'Europe réduisit à l'état de bêtes de somme, un de ces hommes qui naquirent libres, et qui cependant furent vendus comme de vils troupeaux, un Noir aujourd'hui citoyen est aujourd'hui à côté du représentant. Prieur le désigne au peuple, demande après quelques réflexions pleines de sentiments, si on le reconnaît pour frère. Ce mot est à peine achevé que des acclamations affirmatives retentissent de toutes parts et le représentant donne à ce digne Africain l'accolade fraternelle. $»^{65}$

L'examen de la séance du 7 février 1794 au Club des Jacobins pour la liberté générale fragilise la thèse d'un complot antiabolitionniste contre Danton, car il montre un Robespierre assistant à une discussion où tout le monde s'accordait sur la liberté des Noirs dans les colonies françaises et à Santo-Domingo: seule la question de l'indépendance de la colonie espagnole divisait.

En dépit des apparences, cette politique émancipatrice n'épargne pas non plus l'image nouvelle des commissaires de Saint-Domingue, Sonthonax et Polverel dont le décret d'accusation du 16 juillet 1793 a été confirmé le 22germinal an II (11 avril 1794), pour partir le lendemain avec le décret d'abolition. C'était en fait un texte de police, comparable à celui signé le mois précédent par Saint-Just et Collot d'Herbois à l'encontre de Page et Brulley. Il réclamait la destitution du commandant Simondes, chargé de faire arrêter Sonthonax et Polverel, sur demande écrite de Dufaÿ, Mills et Belley ( 8 germinal an II - 28 mars 1794) ${ }^{66}$. Les trois députés y présentaient Simondes comme un dangereux facteur de destabilisation de la colonie du fait de son lourd passé de créole, ennemi des hommes de couleur. Robespierre, signant ce texte, se situait donc dans la philosophie émancipatrice du 4 février 1794. L'arrestation et la mort de Danton et de Delacroix n'empêchèrent donc pas Robespierre à propos de Sonthonax et de Polverel de distinguer le bon grain abolitionniste de l'ivraie brissotine (ou supposée telle). 
En plus de la complémentarité des décrets des 11 et 12 avril 1794 il faut prendre en compte un autre décret rédigé le 3 floréal an II (22 avril 1794) qui confirme ce diagnostic. Sur réclamation écrite de Pierre Chrétien et Victor Hugues, deux commissaires choisis par le ministère de la Marine pour l'exécution du décret dans les Îles-du-Vent, envoyée à Barère et Billaud-Varenne le 26 germinal an II (15 avril 1794) ${ }^{(67)}$, Robespierre co-signa avec Barère, Collot d'Herbois, Carnot, Billaud-Varenne et Prieur de la Côte d'Or, un ordre nommant Sijas, un troisième commissaire de sécurité68. La lecture du décret du 22 avril, confrontée à celle de la lettre du15, indique qu'il s'agissait de surmonter les difficultés d'application géographique causées par une zone composée de trois Îles-du-Vent, Martinique, Guadeloupe, Sainte-Lucie, de prévenir une non-application du décret d'abolition de la servitude des Noirs en cas de décès de l'un d'entre eux ou de différend entre les deux commissaires ${ }^{69}$. Par ailleurs la missive du 12 avril prévoyait l'abolition dans les colonies françaises, toutes explicitement nommées. Et nous ne voyons nul embarras spécifique à propos des Mascareignes, comprises dans le texte, à l'instar de Saint-Domingue, des Iles-du-Vent et de Cayenne. En fait l'embarras commence lorsque le comité suspend le 2 floréal an II (21 avril 1794) le décret pour l'océan Indien. Mais cette fois, Robespierre n'apposa pas son nom au texte comme le firent ses cinq collègues cosignataires du texte du 22 avril. S'agissait-il d'une méfiance personnelle à l'égard d'une possible manœuvre de Benoît Gouly, le député crypto-esclavagiste des Mascareignes, peut-être commanditaire de cette suspension $\left({ }^{70}\right)$ ? Une altercation aux Jacobins entre Robespierre et Gouly le 6 thermidor an II (24 juillet 1794) pourrait étayer cette hypothèse.

35 Mais il serait hasardeux de l'affirmer, sans commettre l'erreur d'oublier le caractère fortuit des signatures, présences et lectures de lettres reçues. Plus encore, nous ne savons rien de la lettre qui a motivé la suspension formulée dans ce décret du 21 avril: des raisons militaires, liées aux risques d'une expédition beaucoup plus lointaine que les Antilles ne sont pas à exclure.

36 Ajoutons-y la complexité de Benoît Gouly. Dans son combat contre Robespierre en thermidor le député créole s'est appuyé sur Tallien, Fouché, Dubois-Crancé et Thuriot $\left({ }^{71}\right)$, soit des Montagnards résolument opposés avant et après Thermidor aux colons esclavagistes de Saint-Domingue $\left({ }^{72}\right)$; à partir de février 1794 , au Comité des colonies, Gouly plaida la libération de Leborgne et l'obtint peu après le 9 thermidor ${ }^{73}$.

En tout cas en avril 1794, Robespierre avait bien changé par rapport au discours de novembre 1793, et à ses Notes contre les Dantonistes. Ou plutôt il renouait avec son approche rigoureuse, dénuée de tout sectarisme, formulée en mai 1792 et peut-être en décembre 1793 par le biais de Desmoulins dans le Vieux Cordelier. Robespierre distingua à nouveau politique métropolitaine et politique coloniale : Sonthonax et Polverel sont suspects d'avoir été de vigoureux Brissotins dans le débat sur la guerre d'attaque en 1791-1792, mais on ne saurait leur reprocher pour autant d'avoir libéré les Noirs de Saint-Domingue. Robespierre était redevenu « le juste » que présentait Labuissonnière en juin 1793.

38 Par ailleurs, le « $\mathrm{n}^{\circ} 5$ " des notes fournies au Comité de salut public, suspecté par Robespierre de Contre-Révolution, contenait dans sa deuxième partie, un autre pamphlet contre Sonthonax et Polverel. Les rapports sur les colons emprisonnés à Brest sous l'autorité de Prieur de la Marne et de Jeanbon Saint-André montrent que l'hostilité aux deux commissaires fut prise en compte dans leur incarcération. Peut-on 
alors affirmer que ces deux hommes ont échappé à la guillotine pour être arrivés après le 9 thermidor? Il serait là aussi risqué de l'affirmer. François Polverel, le fils d'un des commissaires, arrivé de Saint-Domingue en France avant thermidor et arrêté sur ordre officiel du Comité de salut public, y a bien échappé. Mais plus encore, alors qu'il devint dans la période thermidorienne violemment antirobespierriste, il affirma alors que cet ordre d'arrestation... fut faussement attribué au Comité de salut public ${ }^{74}$. Par ailleurs les critiques que Dufaÿ tient sur Robespierre le 13 février 1795, sont plus mesurées que ne l'a affirmé Aimé Césaire ${ }^{75}$. Dufaÿ n'a pas opposé avant le 16 pluviôse l'esclavagisme de Robespierre à l'abolitionnisme de Barère. Les propos rapportés par Barère à Dufaÿ traitaient des divergences relatives à certaines modalités d'application du décret: envoi de troupes et/ou de nouveaux commissaires. En fait la critique de Robespierre par Dufaÿ se limite ici à celle, artificielle et commune à tous les autres thermidoriens, d'un tyran qui a voulu usurper le pouvoir pour le compte de l'Angleterre ${ }^{76}$.

Dans ce discours, Dufaÿ se plaignit en fait de la négligence de la Convention pour les colonies depuis un an. Il constatait ainsi avec amertume que rien n'avait changé depuis la chute du tyran. De son propre aveu les Thermidoriens ne sont pas plus attachés à l'abolition. À cette différence près que la réaction et le démantèlement du mouvement populaire sont allés de pair avec une libération progressive des colons emprisonnés par les décrets ou décisions de sections de l'hiver 1794, qui parlent, écrivent et agissent désormais au grand jour.

En 1794, au vu notamment des papiers de la commission Courtois, Robespierre adopte une attitude antithétique à celle qu'on lui attribue souvent dans ses discours de mai et septembre 1791 : défense des droits des libres de couleur au détriment des esclaves. À ses yeux tous les hommes méritent en soi considération et ceux, Blancs ou Métis, qui seraient ennemis de la liberté et de l'égalité, des "Nègres ci-devant esclaves", paraissent suspects. Aussi les accusations thermidoriennes des colons de SaintDomingue contre Robespierre d'avoir été un agent des trois députés de la colonie paraissent un hommage, ô combien fondé du vice aristocratique à la vertu égalitaire et universaliste!

\section{NOTES}

1.« Rapport fait au nom du Comité de salut public, par le citoyen Robespierre, membre de ce Comité, sur la situation politique de la République », 27 brumaire an II - 17 novembre 1793, Euvres, tome 10, pp.173-174; Aimé CÉSAIRE, Toussaint-Louverture, la Révolution française et le problème colonial, Paris, 1961, p. 185.

2.Aimé CÉSAIRE, op. cit., p.186.

3.Yves BéNot, La Révolution française et la fin des colonies, Paris, la Découverte, 1987, p. 173.

4.Claude WANQUET, La France et la première abolition de l'esclavage 1794-1802: le cas des colonies orientales, Paris, Karthala, 1998, pp.174-175, à partir d'Y. BÉNOT, op. cit., pp. 100-101 et 153.

5.C. WANQUET, op. cit., pp. 168-176. 
6.Jean-Claude HALPERN, « Entre esclavage et liberté: les variations d'un ethnotype dans la France de la fin du xviIIesiècle ", pp.129-138, dans Actes du 123e congrès national des sociétés historiques et scientifiques, Antilles, Guyane, 575 p., p.137, d'après Albert MATHIEZ « Notes de Robespierre contre les Dantonistes » dans Études sur Robespierre, Paris, 1989, p.136. Georges HARDY, « Robespierre et la question noire », Annales Révolutionnaires, 1920; Y. BÉNOT, « Robespierre, les colonies et l'esclavage », dans Robespierre, de la nation artésienne à la République et aux nations, actes du colloque organisé à Arras le 1er,2 et 3 avril 1993, par l'Université Charles de Gaulle, Lille III, Centre d'Histoire de la région du Nord et de l'Europe du Nord-Ouest, coll. Histoire régionale de Picardie, 1994, pp. 409-421, p.420.

7.Yves BÉNOT, « Un antiesclavagiste kleptomane? En marge de l'affaire Milscent », XVIIIesiècle, n²2, 1990, pp. 295-300; « Quelques remarques sur les problèmes de l'histoire de la Révolution française dans ses rapports avec la question coloniale ", dans Recherches sur la Révolution, La Découverte, pp. 439-444. Les remarques de Saint-André proviennent de l'ouvrage de Ruth NECHELES, The abbé Grégoire, The odyssey of an Egalitarian, 1787-1831, Westport Connecticut, 1971, pp.131 et 134.

8.J.-D. PIQUET, «L'arrestation de Sonthonax et Polverel: Robespierre réceptionne une lettre des trois députés de Saint-Domingue contre un créole suspect, avril 1794 », AHRF $\mathrm{n}^{\circ}$ 306, octobre-décembre 1996, pp.713-717 et erratum n³09, juillet-septembre 1997, pour le texte complet et corrigé de Dufaÿ, Mills et Belley. « Le Comité de salut public et les fêtes sur la liberté des Noirs, Châlons-sur-Marne, Lyon, Paris (L'Être Suprême) ", Robert-Louis STEIN, Sonthonax, the Lost Sentinel of the Republic, Farleight Dickison University Press, London and Toronto, 1984, pp.111-112; Yves BÉNOT, « Robespierre, les colonies ", art. cit. p. 420.

9.Cyrille Lionel R. JAMES, Les Jacobins noirs, Paris, 1938, Éditions Caribéennes, 1983. 10.Florence GAUTHIER, Triomphe et mort du droit naturel en révolution,1789-1795-1802, Paris, PUF, 1992. «La Révolution française et le problème colonial: le cas Robespierre », AHRF, avril-juin 1992 pp.169-192; « Le rôle de la députation de Saint-Domingue dans l'abolition de l'esclavage ", dans Les abolitions de l'esclavage de Léger-Félicité Sonthonax, 1793, 1794-1848, Presses universitaires de Vincennes UNESCO, 1995, pp.199-211. 11.J.-D. PIQUET, « L'émancipation des Noirs dans la pensée et le processus révolutionnaire français (1789-1795) », Doctorat N.R., Paris VIII, octobre 1998, 736 p. 12.F. GAUTHIER, op. cit., pp. 216-220.

13.Ibid.

14.Yves BÉNOT, « Un antiesclavagiste kleptomane? », art. cit, pp.297-298.

15.Claude WANQUET, op. cit., pp. 33-34.

16. Yves BÉNOT, art. cit.

17.AN, F74434, Dossier « Danton »; François CHEVREMONT, Marat, esprit politique accompagné de sa vie scientifique, politique et privée, Paris, 1880, 2 vol, tome 2, annexes. Précis de la Vie de Roume par lui même, 17 septembre 1795, AN, AF II 302; F. GAUTHIER, « La Révolution française... », art. cit. pp. 176-177.

18.P. R. ROUME DE SAINT-LAURENT, Sur la question des gens de couleur, 11 mai 1791, BNF, 4 lk9 141.

19. , tome 68 , p. 307.

20.AN, DXXV 76, Chabot l'expliquait ainsi à Page et Brulley venus lui demander d'annuler la libération.

21.AN, D XXV 56, dossier Roume. 
22.Voir Archives parlementaires, 30 janvier 1793, tome 58, pp. 67-87. « Rapport de Philippe Rose-Roume sur la situation à Saint-Domingue en qualité de commissaire civil », pp. 81-82, « Extrait du réquisitoire de M.Larchevesque-Thibault, procureur syndic de la commune du Cap », 1er août 1792; « Lettre du citoyen LarchevesqueThibault, procureur-syndic de la commune du Cap », p. 83.

23. Ce personnage est très discrètement sorti de l'obscurité, à l'occasion du centcinquantenaire de la seconde abolition. Son nom est signalé dans la chronologie détaillée de l'exposition parlementaire des deux abolitions comme le premier homme de couleur à avoir siégé à la Convention en septembre 1793.

24.AN, DXXV 76 dos 751.

25. , tome 73, séance du 3 septembre 1793, pp.354-355.

26.Joseph LEBORGNE, Enfin la vérité sur les colonies en réponse à Janvier Littée député de couleur, 24 vendémiaire an III- 15 octobre 1794 (p. 29 d'après le rapport de Garran), B.H.V.P., 604.352.

27.27, 29 brumaire an II - 17, 19 novembre 1793, AN, DXXV 76 dos 753.

28.Sophie WAHNich, L'étranger dans la Révolution, Paris, Albin Michel, 1997, pp. 195-197.

29. Le défenseur de la Constitution, $\mathrm{n}^{\circ} 3-31$, mai 1792, in Robespierre, Euvres, tome 4, p.84; Voir Y.BÉNOT, « Robespierre, les colonies... », art. cit. pp. 413-414; J.-D. PIQUET, op. cit. 30.Jean-Paul BERTAUD, Camille et Lucille Desmoulins, un couple dans la tourmente, Paris, 1986, p.142.

31.Le Vieux Cordelier, $\mathrm{n}^{\circ}$ 2, 20 frimaire an II - 10 décembre 1793, Paris, Belin, 1987, 153 p., p. 45 .

32.F. GAUTHIER, op. cit., p. 224; Marc BELISSA, Fraternité universelle et intérêt national 1713-1795. Les cosmopolitiques du droit des gens, Paris, Kimé, 1998. J.-D. PIQUET, « La déclaration constitutionnelle de paix à l'Europe, grand sujet de débat dans la Révolution de 1791 à 1794 ", 119e congrès du C.T.H.S., Amiens, 1994, La Révolution française, la guerre et la frontière, sous la direction de Monique Cubells, Paris, éd. du C.T.H.S., 2000, 527 p., pp.387-397.

33.Papiers inédits...trouvés chez Robespierre, Saint-Just, Payan, etc., Paris, 1828, tome 2, pp. 176-178. B.H.V.P., 8 H 92.

34.Archives parlementaires, tome 84, séance du 15 pluviôse an II - 3 février 1794. Le député Camboulas se félicita de la nouvelle présence à la Convention d'un Noir, d'un Jaune et d'un Blanc. F.GAUTHIER, Triomphe et mort..., p.234.

35.Papiers inédits..., op. cit., tome 3, p. 55.

36., tome 3, pp.170-173.

37.Leborgne à Janvier Littée, BNF, 8 lk12 326 vendémiaire an III - 27 septembre 1794; Janvier LITTÉE, Réponse à P.-J. Leborgne, BNF, 8 lk9 218, 17 vendémiaire an III - 8 octobre 1794.

38. Archives parlementaires, tome 84 , annexes III au $n^{\circ} 36$ de la séance du 10 pluviôse an II - 29janvier 1794.

39.F. GAUTHIER, « Inédits de Belley, Mills et Dufaÿ, députés de Saint-Domingue, de Roume et du Comité de salut public, concernant le démantèlement du réseau esclavagiste en France (février-mars1794) », AHRF, n 302, octobre-décembre 1995, pp. 607-611.

40.Débats entre les accusateurs et les accusés dans l'affaire des colonies, BNF, 8 lb38 1662, 9 vol, tome2, 2 ventôse an III - 20 février 1795, p. 283.

41.AN, DXXV 76 dossier 755.

42.AN, F7/4765 Dos 3. 
43.Ibid.

44.AN F7 4774 46, « Thomas Millet » plainte contre Duffay (sic), J.-D. PIQUET, « Le Comité de salut public... », art. cit.

45.Millet dans le même texte fournit un portrait mesuré de l'abbé Grégoire qu'il rencontra en février 1791. Il oublie justement qu'il lui demandait de renoncer à la défense des libres de couleur: «Lettre à Grégoire ", Annales politiques, civiles et littéraires du XVIIIesiècle, 1791, p.399.

46.Y. BÉNOT, « Robespierre, les colonies... ", art cit. p.420; C.WANQUET, op. cit., pp.170-171. 47.Selon Y. BÉNOT, « Comment la Convention a-t-elle aboli l'esclavage en l'an II? », Révolutions aux colonies, AHRF, 1993. Le 16 pluviôse, " au nombre de sept, BillaudVarenne, Saint-Just et Prieur de la Marne étant en mission, Couthon malade, les membres du Comité recevaient Page et Brulley, écoutaient leurs doléances. Il est vrai que le Comité ne leur accorde pas la confrontation demandée qui ferait perdre trop de temps, mais est prêt à accueillir leurs mémoires et à les examiner ». En fait Billaud partit en mission le 29 pluviôse (17 février) et peut donc à la date du 4 février être adjoint aux six autres; Robespierre était occupé par la préparation du rapport sur les principes de morale politique qui guident la Convention nationale sur son administration intérieure qu'il présenta le 17 pluviôse ( 5 février). De son côté, Couthon n'était pas encore tombé malade: le 4 février, d'après leur registre Page et Brulley se sont présentés à son domicile et ne l'ont pas trouvé. Il aidait sans doute Robespierre à la rédaction du rapport; le 5, à la Convention, Couthon demanda là-dessus la mention honorable.

48.PAGE, BRULLEY, Calomniateurs dénoncés à la Convention nationale, 8 fructidor an II - 25 août 1794, BNF, 8 lk12 460.

49.Débats entre les accusateurs et les accusés... , op. cit., pp.282-283.

50.ROBESPIERRE, Papiers inédits, tome 1, pp.376-378, pièce n XXVIII (version de 1824).

51.PAGE, BRULLEY, Notes fournies au Comité de salut public, BNF, $8 \mathrm{lk} 12$ 452. Ces notes traitaient de la situation à Saint-Domingue en 1793, revue et corrigée par leurs auteurs. 52.Page et Brulley y ajoutèrent cependant l'exclamation « Enfin! ».

53.AN, DXXV 68, Dos 682.

54.AN, Police générale F7 4670 Dos 3, « De Ragis ».

55.AN, DXXV 72, dos 720, p. 17. Il ne s'agissait pas de Saint-André, mais de Saint-Just, qu'ils avaient certes également beaucoup fréquenté, mais dont ils ignoraient le rôle décisif dans leur arrestation. Page et Brulley en juin 1794 se sont simplement félicités de l'annonce de son retour.

56.Albert MATHIEZ, op. cit., p. 136.

57.Jean-Claude HALPERN, art. cit., p. 137. Georges HARDY, art. cit.

58. Albert MATHIEZ, op. cit.

59.Jules MICHELET, Histoire de la Révolution, Paris, Gallimard, Pléiade, 2 vol, tome 2, p. 775.

60.Ibid., p. 771.

61.Y. BÉNOT, « Robespierre, les colonies... », p. 420, « Comment la Convention... », art.cit., p. 13.

62.Pierre CARON, Paris sous la Terreur, tome 4, p. 7, rapport du 21 pluviôse an II - 9 février 1794.

63.Le Journal de la Montagne, Le Mercure universel, Le Républicain, Le Messager du Soir, Le Créole patriote et sous réserve de vérification Le Journal du Soir et du Matin de Sablier. 
64.C. MAZAURIC, Présentation des CEuvres de M. Robespierre en 10 vol, réimpression en facsimilé, éd. Phénix, 2000, 16 p., pp. 9-10 à propos de sa présence supposée à la Convention le 17 pluviôse (sa présence le 16 étant, on l'a vu, très improbable). 65.Le Journal de la Montagne, $\mathrm{n}^{\circ}$ 74, 22 messidor an II - 10 juillet 1794.

66.AN, DXXV 57, dos 565 ter., J.-D. PIQUET, « L'arrestation de Sonthonax et Polverel... », art cit.

67.AN, F74435 plaquette 3, pièces 88 et 90 .

68.Ibid, pièce 74 et A. AULARD, Recueil des actes du Comité de salut public, tome 12, p.709.

Mais faute d'avoir reçu l'ordre avant l'embarquement, Sijas ne put partir: A. AULARD, op. cit., tome 13, pp. 84 et 671.

69.Le cas de Hugues, créole esclavagiste de Saint-Domingue, dénonciateur de Roume à Rochefort en juin 1793 et de la députation de Saint-Domingue en janvier 1794 à Lorient, posait peut-être problème à Chrétien issu d'un milieu sans-culotte de la Dordogne, et qui avait été à ce titre nommé à l'automne 1793 au côté de Sijas et d'un certain Bétrine. En mars 1794, Bétrine fut mystérieusement remplacé par Hugues tandis que Sijas fut oublié.

70.Y. BÉNOT, « Quelques remarques sur les problèmes... », art. cit., p. 439.

71.F. GAUTHIER, Triomphe et mort..., op. cit., p. 261.

72.J.-D. PIQUET, op. cit., p. 634.

73.Ibid. et AN, DXXV 56 dos Leborgne et DXXV 77 dos 762. Leborgne le rappela dans ses brochures: Leborgne à Janvier Littée, Enfin la vérité sur les colonies, op. cit.

74.Nicolas ROCHE, « La question coloniale en l'an III: l'invention du mythe de la Gironde ", AHRF, n³02, pp.587-605, octobre-décembre 1995, p. 593.

75.Aimé CÉSAIRE, op. cit., p. 185.

76.Voir F.GAUTHIER, « Note sur le système de défense de Dufaÿ sous la Convention thermidorienne », Triomphe et mort... pp. 267-269.

\section{RÉSUMÉS}

La position de Robespierre sur le décret du 16 pluviôse an II (4 février 1794) relatif à l'émancipation des esclaves, a été controversée. Il a pourtant bien agi pour l'application de celuici. Certains propos formulés en novembre 1793 ont accrédité en lui une réputation d'esclavagiste. Mais on peut les expliquer par la présence d'un député mulâtre de la Martinique, Janvier Littée, très lié aux colons blancs de Saint-Domingue. Or ce député et ces liens, Robespierre les surveillait peu avant sa mort, avec l'aide de la section parisienne de Bonne-Nouvelle qui avait anticipé de plusieurs semaines la loi d'arrestation des colons hostiles au décret et prouvé ainsi l'importance d'un mouvement populaire. Ce mouvement populaire agissant de concert avec les Robespierristes s'était déjà manifesté à Lorient en janvier 1794. Par ailleurs Robespierre a également signé au Comité de salut public en avril 1794 deux ordres d'application du décret du 4 février.

Robespierre and the liberation of blacks in the Year II from the archives of the committees and the papers of the Courtois Commission. 
Although he ordered the application of the decree of 16 pluviose YearII (4February 1794) which suppressed slavery, Robespierre's position on the emancipation of the slaves has been steeped in controversy. Based on a few statements he made in November, 1793, Robespierre is reputed to have supported slavery. Those statements, though, reflected the influence, at the time, of Janvier Littee, a mulatto deputy from Martinique, who was closely connected to white colonists in SaintDomingue. However, a short time before his death at the end of July, 1794, Robespierre, with the assistance of activists from the Bonne-Nouvelle section, monitored the activities of Littee and his colonial allies. Prior to that, and several weeks before the decree's issuance, the Bonne-Nouvelle section arrested some colonists who were hostile to emancipation, thus establishing the importance of the popular movement to abolitionism. In January, 1794, this popular movement was acting in concert with Robespierrists at Lorient. Moreover, in April, 1794, Robespierre, on behalf of the Committee of Public Safety, signed two administrative orders into law relative to the 4 February decree.

\section{INDEX}

Mots-clés : Robespierre, 1re abolition de l'esclavage colonial, mouvement populaire, commission Courtois, comités de gouvernement

\section{AUTEUR}

JEAN-DANIEL PIQUET

APECE 